\title{
EPR spectra of spin labels in lipid bilayers. II. Rotation of steroid spin probes
}

Hansgeorg Schindler and Joachim Seelig

Citation: The Journal of Chemical Physics 61, 2946 (1974); doi: 10.1063/1.1682437

View online: http://dx.doi.org/10.1063/1.1682437

View Table of Contents: http://aip.scitation.org/toc/jcp/61/7

Published by the American Institute of Physics

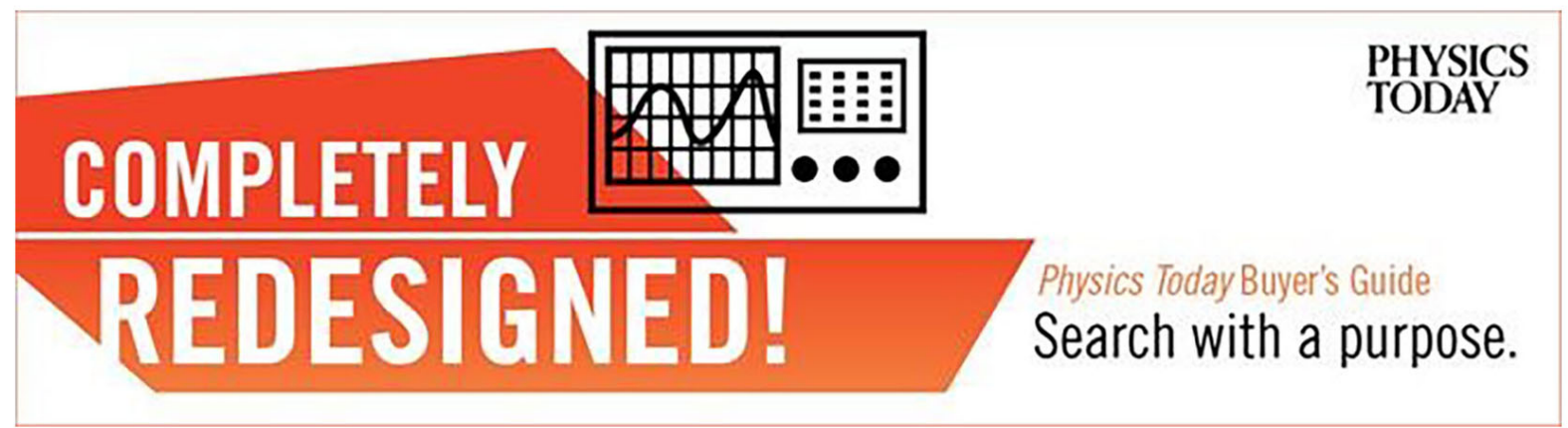




\title{
EPR spectra of spin labels in lipid bilayers. II. Rotation of steroid spin probes*
}

\author{
Hansgeorg Schindler and Joachim Seelig \\ Biocenter of the University of Basel, Department of Biophysical Chemistry, Klingelbergstrasse 70, Ch-4056 Basel, Switzerland \\ (Received 15 April 1974)

\begin{abstract}
The spin label method is used to investigate the nature of molecular motion in a liquid crystalline bilayer. The EPR spectra of different spin labels are simulated by means of perturbation theory. Using the diffusion model with a restoring potential the rotational diffusion coefficients for the different spin labels are evaluated from the linewidth. Rotational diffusion and viscosity are related by a modified Stokes-Einstein-Perrin formula. The correction factors account for the difference between the effective viscosity during rotation and the (translational) viscosity and are interpreted in terms of the geometry and the ordering of the spin probes. The bilayer viscosity $\eta$ is found to be $3.7 \mathrm{cp} \leq \eta \leq 5.7 \mathrm{cp}$.
\end{abstract}

\section{INTRODUCTION}

EPR spectra of spin probes in liquid crystalline bilayers can be analyzed with high precision using perturbation theory. 'The physical parameters entering the theory are the order parameters $S_{i}$ and the correlation times $\tau_{2 m}$. The order parameters are determined from the position of the resonance lines and they describe the anisotropy of the molecular motion. The correlation times are evaluated from the linewidth and are related to the rates of spin probe rotation.

Our previous approach was based on the strong collision model for molecular reorientation. In this model the physical interpretation of the correlation times is difficult. We have now calculated the spectral densities by means of the Brownian diffusion model and the linewidths are now related directly to the principal components $R_{i 1}$ of the molecular diffusion tensor.

The experimental parameters $S_{i}$ and $R_{i i}$ depend on the structure of the bilayer as well as on the geometry of the spin probe. By comparing spin probes of different geometry it should be possible to extract information about the properties of the bilayer itself. In the following this is illustrated for the viscosity $\eta$, which is a translational property of the bilayer. It is shown that $\eta$ can be related to the rotational motions of the molecules in the bilayer. Therefore a linewidth analysis of the spin probes provides information about both the rotational and the translational diffusion of the molecules in a lipid bilayer. Since only very low concentrations of spin probes are needed, this method is an alternative to the spin exchange method ${ }^{2-6}$ for measuring translational diffusion.

Our analysis proceeds as follows: (1) We simulate the EPR spectra of steroid spin probes dissolved in a bilayer and investigate the effect of various models describing the molecular reorientation. (2) Since a molecule is subject to a different effective viscosity during rotation than during translation, we discuss the necessary correction factors for the commonly used Debye model to relate rotational diffusion to translational viscosity. (3) Taking into account the molecular geometry of the spin probes we interpret the experimental diffusion coefficients $R_{i i}$ in terms of a bilayer viscosity $\eta$.

\section{EXPERIMENTAL}

The lipid bilayer employed in our investigation is a smectic liquid crystal with the following chemical composition: decanol (42 wt. \%), sodium decanoate (28 wt.\%) and water ( $30 \mathrm{wt} . \%$ ). To study the diffusion and viscosity properties of this phase we used the nitroxide spin labels shown in Fig. 1. The experiments were carried out at label concentrations lower than 0.1 wt. \%, so that dipole-dipole and spin-spin relaxation mechanisms could be neglected for the linewidth calculation. Single crystal data are available for the steroid label II in Fig. 1, but they refer to an environment which is different from that in a liquid crystalline phase. A further complication arises from the fact that the interaction tensors are diagonalized by another set of coordinate axes than the anisotropic diffusion tensor. The Eulerian angles $\left(\alpha_{1}, \beta_{1}, \gamma_{1}\right)$ relating the two coordinate systems can roughly be estimated by inspection of molecular models. Starting with single crystal data and estimated Eulerian angles we established for each spin label a slightly corrected set of these constants (Table I) so that all EPR spectra could be simulated with the same accuracy. The EPR spectra are determined by essentially four molecular parameters, namely $S_{3}, S_{1}-$ $S_{2}, R_{\| 1}$, and $R_{1}$. In principle, all parameters can be obtained from one experimental spectrum. Higher accuracy is achieved by comparing the EPR spectra arising from different sample geometries. The bilayers were therefore oriented between quartz plates and the magnetic field applied parallel and perpendicular to the bilayer normal. A random (isotropic) distribution of bilayer regions was also investigated. Experimental and theoretical EPR spectra characteristic of the three types of orientation are shown in Figs. 2-4. All spectra were simulated with the same set of molecular parameters assuming Lorentzian lineshapes.

In addition to the motional modulations of the hyperfine and Zeeman interactions the linewidth is also dependent on unresolved proton hyperfine splittings and spin rotation interactions. This residual linewidth $X$ was approximated by ${ }^{1,7}$

$$
X=b+c \cos ^{2} \beta^{\prime} \text {. }
$$

The parameters $b$ and $c$ are fairly constant for a given 


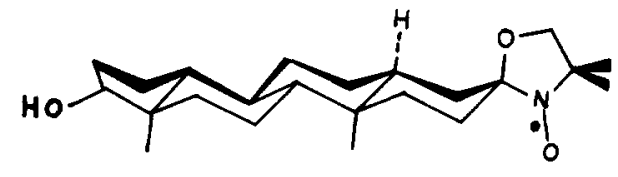

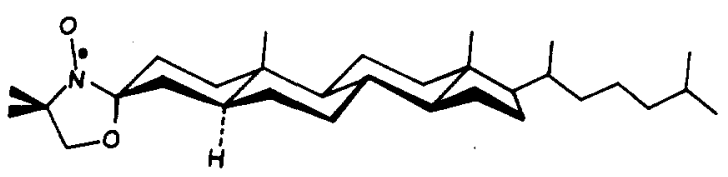

FIG. 1. Steroid nitroxide labels I and II.

spin probe.

It is rather difficult to obtain a perfectly planar orientation of bilayer domains. The inhomogeneity of the sample ordering was taken into account by assuming a Gaussian distribution function for the director axes. ${ }^{1}$ For all spectra the spread angle $\vartheta_{0}$ was found to be $\vartheta_{0}$ $\approx 4^{0}$. The measurements were made at room temperature $\left(\approx 22^{\circ} \mathrm{C}\right)$ with a Varian $E-9$ spectrometer $(\sim 9.35$ $\mathrm{GHz}$ resonance frequency).

\section{SIMULATION OF SPIN PROBE SPECTRA}

The position and the width of the resonance lines are calculated essentially as described previously. ${ }^{1}$ We have introduced two changes. Firstly, the spatial factors $F_{\lambda}^{(l,-m)}$ are expressed in the coordinate system of the molecular diffusion tensor. This is easily done by
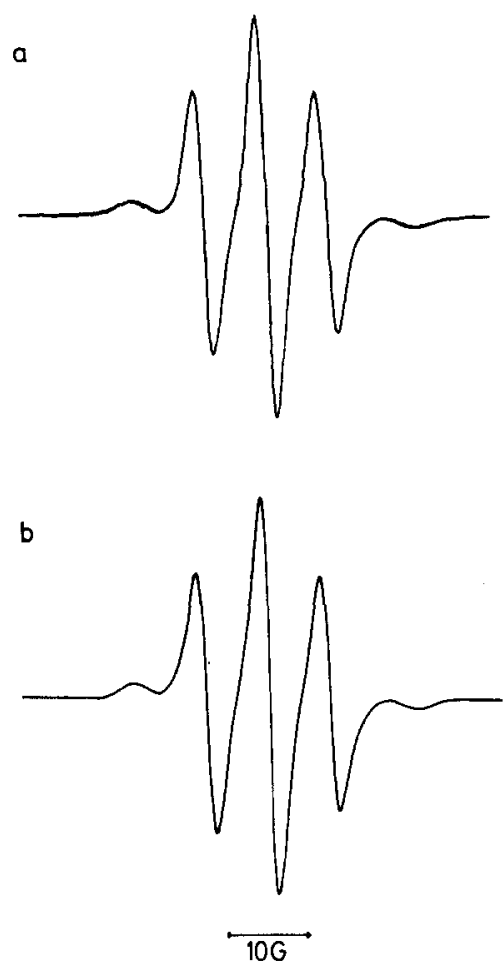

FIG. 2. Steroid label $I$ in a planar oriented sample of bilayers at $24^{\circ} \mathrm{C}$. Magnetic field parallel to bilayer normal. (a) $\mathrm{Ex}-$ perimental spectrum, (b) Calculated spectrum with $S_{3}=0.626$, $S_{1}-S_{2}=0.09, R_{11}=8.3 \times 10^{8} / \mathrm{sec}, R_{1}=1.0 \times 10^{8} / \mathrm{sec}$, spread angle $\vartheta_{0}=4^{\circ}$, residual line width $b=1.3 \mathrm{G}, c=0 \mathrm{G}$.
TABLE I. Ir reducible tensor components (expressed in the coordinate system of the nitroxide group).

\begin{tabular}{lcc}
\hline & steroid I & steroid II \\
\hline$g$ & 2.0055 & 2.0055 \\
$\Delta g$ & $-1.0 \times 10^{-3}$ & $-1.0 \times 10^{-3}$ \\
$\delta g$ & $1.5 \times 10^{-3}$ & $1.5 \times 10^{-3}$ \\
$a$ & $13.8 \mathrm{G}$ & $14.7 \mathrm{G}$ \\
$\Delta a$ & $8.1 \mathrm{G}$ & $8.6 \mathrm{G}$ \\
$\delta a$ & $0.8 \mathrm{G}$ & $0.8 \mathrm{G}$ \\
$\left(\alpha_{1}, \beta_{1}, \gamma_{1}\right)$ & $\left(90^{\circ}, 80^{\circ}, 70^{\circ}\right)$ & $\left(90^{\circ}, 80^{\circ}, 70^{\circ}\right)$ \\
\hline \hline
\end{tabular}

replacing the $F_{\lambda}^{(1,-m)}$ in Ref. 1 by

$$
F_{\lambda}^{(l,-m)}-\sum_{n} D_{m, n}^{(l)}\left(\alpha_{1}, \beta_{1}, \gamma_{1}\right) F_{\lambda}^{(l,-n)} .
$$

The nitrogen $2 p \pi$ orbital of the steroid labels is oriented perpendicular to the long molecular axis and the Eulerian angles become $\alpha_{1}=90^{\circ}, \beta_{1}=80^{\circ}$, and $\gamma_{1}=70^{\circ}$. Secondly, we have investigated the effect of various correlation functions on the line shape simulation. This change in the spectral densities is more important, because it bears on the nature of the molecular motions in the liquid crystalline phase. In our previous work the molecular reorientation was described using the strong collision model. The conditional probability in this model is given by

$$
P\left(\Omega_{0} / \Omega, t\right)=\delta\left(\Omega_{0}-\Omega\right) e^{-t / \tau}+P_{0}(\Omega)\left(1-e^{-t / \tau}\right),
$$

so that the correlation function takes the form

$$
\begin{aligned}
& \overline{\left[D_{p, m}^{*(2)}(\Omega)-\overline{D_{p, m}^{*(2)}(\Omega)}\right]\left[D_{p^{\prime}, m^{\prime}}^{(2)}\left(\Omega_{0}\right)-\overline{D_{p^{\prime}, m^{\prime}}^{(2)}\left(\Omega_{0}\right)}\right]} \\
& \quad=\left[\overline{D_{p, m}^{*(2)}\left(\Omega_{0}\right) D_{p^{\prime}, m^{\prime}}^{(2)}\left(\Omega_{0}\right)}-\overline{D_{p, m}^{*(2)}\left(\Omega_{0}\right)} \overline{D_{p^{\prime}, m^{\prime}}^{(2)}\left(\Omega_{0}\right)}\right] \cdot e^{-t / \tau_{2 m} .} .
\end{aligned}
$$
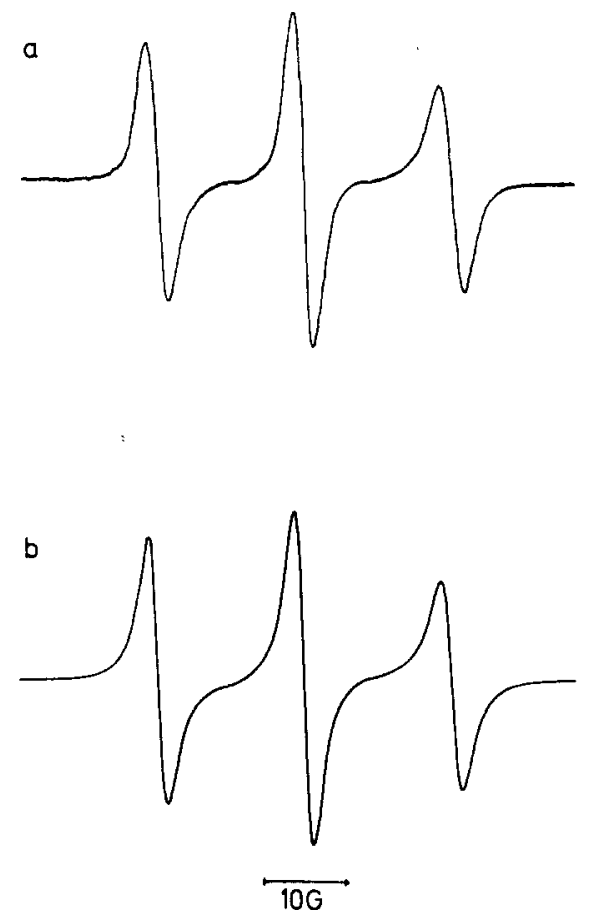

FIG. 3. Steroid label I in a planar oriented sample of bilayers at $24^{\circ} \mathrm{C}$. Magnetic field perpendicular to bilayer normal. (a) Experimental spectrum, (b) Calculated spectrum. Same parameters as in Fig. 2. 

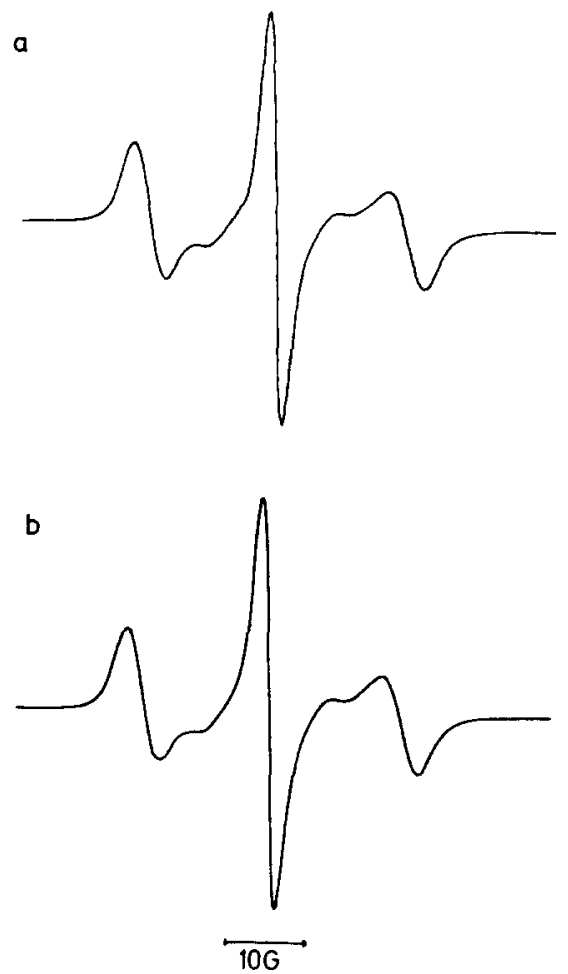

FIG. 4. Steroid label I in random distribution of bilayers at $24^{\circ} \mathrm{C}$. (a) Experimental spectrum, (b) Calculated spectrum. Same parameters as in Fig. 2.

The bar indicates averages over the Boltzmann distribution function $P_{0}(\theta) \sim \exp [-V(\theta) / R T]$, where the restoring potential $V(\theta)$ is approximated by

$$
V(\theta)=q \cos ^{2} \theta \text {. }
$$

A quite different model is the Debye model of rotational Brownian motion. For the diffusion of a symmetric top molecule in the absence of a restoring potential the relations between correlation times $\tau_{2 m}$, rotational diffusion $R_{(11 / 1)}$ and viscosity $\eta$ take the well known forms

$$
\tau_{2 m}^{-1}=6 R_{\perp}+m^{2}\left(R_{11}-R_{1}\right), \quad R_{(1) \perp)}=k T /\left(8 \pi \eta f_{(111)}\right) .
$$

$f_{11}$ and $f_{\perp}$ are geometric factors which depend on the molecular shape only. ${ }^{8}$ For the diffusion in a liquid crystalline phase the situation is more complicated because the molecules are subject to the ordering potential $V(\theta)$. The diffusion is thus influenced by the interaction potential and the calculation of the needed spectral densities is mainly a problem of solving the rotational diffusion equation with a cylindrically symmetric restoring potential. This problem was first treated by Nordio et al. ${ }^{9}$ and more recently by Polnaszek et al. ${ }^{7}$ and by Luckhurst et al. ${ }^{10}$ Using Nordio's numerical approach we therefore calculated the spectral densities as a function of the diffusion ratio $\left(R_{\mathrm{l}} / R_{1}\right)$ and of the order pa-

TABLE $\Pi$. Rotational diffusion coefficients, order parameters, and effective viscosities of spin labels dissolved in a liquid crystalline bilayer at $22^{\circ} \mathrm{C}$.

\begin{tabular}{lllllll}
\hline \hline Spin Label & $R_{\perp}\left[10^{8} \mathrm{sec}^{-1}\right]$ & $R_{\mathrm{tt}}\left[10^{8} \mathrm{sec}^{-1}\right]$ & $S_{3}$ & $S_{1}-S_{2}$ & $\eta_{11}^{\text {oft }[\mathrm{cp}]}$ & $\eta_{1}^{\text {oft }}[\mathrm{cp}]$ \\
\hline Steroid I & $0.98 \pm 0.08$ & $7.8 \pm 0.7$ & 0.65 & 0.09 & 3.6 & 10.2 \\
Steroid Il & $1.39 \pm 0.12$ & $7.6 \pm 0.7$ & 0.55 & 0.09 & 3.7 & 7.2 \\
\hline \hline
\end{tabular}

TABLE III. Correction factors $\boldsymbol{x}_{11}$ and $k_{1}$ for spin labels dissolved in a liquid crystalline bilayer at $22^{\circ} \mathrm{C}$.

\begin{tabular}{lll}
\hline \hline Spin Label & $\mathbf{x}_{\|}$ & $k_{1}$ \\
\hline Steroid I & $0.63-0.98$ & $1.8-2.8$ \\
Steroid II & $0.65-1.00$ & $1.3-1.9$ \\
\hline
\end{tabular}

rameter $S_{3}$. [ $\left[S_{3}\right.$ is directly related to the ordering potential $V(\theta)$. The theoretical spectra calculated with these spectral densities are shown in Figs. 2-4. The same spectra were also simulated with the strong collision model. The results of the two methods can be compared as follows:

(1) For small order parameters $\left(S_{3}<0.5\right)$ the experimental spectra of all spin probes can be described equally well with either the diffusion or the strong collision model.

(2) For steroid spin probes with order parameters in the range $0.5 \leqslant S_{3}<0.75^{11}$ both models show small deviations from the experimental results, but it is not possible to give priority to one of these models. A similar conclusion has been reached for the motion of a steroid spin probe in a nematic liquid crystal. ${ }^{10}$

The Debye model has the advantage of a straightforward molecular interpretation of the parameters. In Table II we therefore summarize the results of the spectral simulations using the Debye model.

\section{ROTATIONAL DIFFUSION AND BILAYER VISCOSITY}

The Debye-Perrin relations (Eq. 2) have been derived for a large particle moving in a homogeneous fluid. In a molecular system where the size of a solute molecule is comparable to that of the solvent molecules the assumptions of the Debye-Perrin theory are no longer valid. Various corrections have been proposed. Here we consider two effects: (1) The rotations of a symmetric top molecule around axes perpendicular to the symmetry axis are hindered by the finite size of the neighboring molecules, thus giving rise to an additional resistive force. ${ }^{12}$ This can be accounted for by increasing the local viscosity by a correction factor $k_{1} \geqslant 1$. (2) On the other hand, inertial or free rotational effects lead to a decrease of the effective viscosity. ${ }^{13}$ The correction factor for this rotational slip is $æ_{(I 1)}$, where $æ$ lies in the range of $0<æ \leqslant 1$. The parameter $æ$ is a measure of the anisotropy of the intermolecular interactions. In a liquid crystal where strong interactions occur if a rod-like molecule is rotated perpendicular to the long molecular axis $æ_{\perp}$ is approximately unity, while $\mathfrak{x}_{11}$ is much smaller than unity. The effective viscosity $\eta^{\text {eft }}$ sensed by a steroid spin probe in a lipid bilayer can thus be written:

$$
\begin{aligned}
& \eta_{11}^{\text {ef }}=æ_{11} \eta, \\
& \eta_{\perp}^{\text {eff }}=k_{1} \eta .
\end{aligned}
$$

With $0<\mathfrak{x}_{\|} \leqslant 1$ and $k_{\perp} \geqslant 1$ it follows 


$$
\eta_{11}^{\text {off }} \leqslant \eta \leqslant \eta_{1}^{\text {etf }} \text {. }
$$

The rotational diffusion coefficients are then given by

$$
R_{(11)}=k T /\left(8 \pi f_{(11)} \eta_{(11)}^{\text {erf }}\right) \text {. }
$$

We use this relation to discuss the results of the spectral simulations. The shape of the steroid spin probe can be approximated by a prolate ellipsoid. From inspection of molecular models the short axis $r_{\perp}$ of this ellipsoid is found to be $r_{1}=3.0 \AA$, while the long axis is $r_{11}=8.4 \AA$ (for both steroids). The geometric factors are then calculated to be $f_{11} \approx 163 \AA^{3}$ and $f_{1} \approx 57 \AA^{3}$, leading to the effective viscosities $\eta^{\text {off }}$ listed in Table $\Pi$.

As expected from the shape of the steroid spin probes both molecules have the same rotational diffusion constants $R_{n}$ and thus sense the same effective viscosity $\gamma_{11}^{\text {etf }}$ for the rotation around the long molecular axis. The corresponding results for rotations perpendicular to this axis deviate from each other. The discrepancy is most likely due to the different loci of the center of gyration. Both labels are anchored in the lipid-water interface and this shifts the center of gy ration towards the boundary region. The more the center of gyration approaches the lipid-water boundary, the larger becomes the resistive force parameter $k_{\perp}{ }^{14}$ This effect must be more pronounced for spin probe I than for spin probe II, since the hydroxyl group in I is a stronger anchoring group than the nitroxide moiety in II.

Using the inequality (7) allows a first estimate of the bilayer viscosity from the steroid data

$$
3.7 \mathrm{cp} \leqslant \eta \leqslant 7.2 \mathrm{cp} \text {. }
$$

If the shift of the center of gy ration is taken into account (using a model similar to that of Shimizu) this interval can be narrowed to

$$
3.7 \mathrm{cp} \leqslant \eta \leqslant 5.7 \mathrm{cp} \text {. }
$$

This leads to the correction factors listed in Table III. The rotational slip factors $x_{\|}$are practically identical for both steroids. The theory predicts that inertial motions should be quenched less rapidly if the system is well ordered. ${ }^{13}$ This is confirmed at least qualitatively by experiments with steroid II in a nematic liquid crystal. ${ }^{10}$ Here the order parameter approaches 0.9 , while the rotational slip factor is reduced to $x_{11} \approx 0.06$. On the other hand the resistive force factor $k_{\perp}$ is larger for steroid I than for steroid II. This is due to the shift of the center of gy ration as explained above.

Using a fatty acid spin label, which shows completely different EPR spectra, we have previously estimated a bilayer viscosity of $\eta=4.8 \mathrm{cp}^{1}$ The precision of the data is probably not better than $\pm 1 \mathrm{cp}$ but the agreement of the different experiments indicates that the translational viscosity should be of the order of $5 \mathrm{cp}$.

This result is supported by more direct measurements of translational diffusion coefficients in related liquid crystalline systems. If the molecules constituting the bilayer (decanol, decanoic acid) are approximated by rotation ellipsoids of $10 \AA$ length and $4.6 \AA$ diameter their translational diffusion coefficient is found to be

$$
D_{\text {trans }}^{22^{\circ}} \approx 1.3 \times 10^{-6} \mathrm{~cm}^{2} \mathrm{sec}^{-1} \text {. }
$$

This agrees very well with NMR pulse measurements by Roberts of the lamellar phase of potassium laureate, where a self diffusion coefficient of $2.4 \times 10^{-6} \mathrm{~cm}^{2} \mathrm{sec}^{-1}$ (at $80^{\circ} \mathrm{C}$ ) was found. ${ }^{15}$ Similar diffusion coefficients have been measured for the hexagonal ${ }^{15}$ and the cubic ${ }^{16}$ phase of the same system.

In conclusion, our results indicate that the properly corrected Debye-Perrin model is well suited to describe the EPR spectra and the rotational motion of various spin labels in a lipid bilayer. This approach is not limited to simple soap-like liquid crystals, but can also be applied to phospholipid bilayers or biological bilayer membranes. However the viscosity $\eta$ in these systems is at least by a factor of 10 larger than in the examples discussed above. The line shape analysis may then become more complicated, since the application of perturbation theory may no longer be justified. ${ }^{17}$

*Supported by the Swiss National Science Foundation under Grant No. 3.8620.72.

${ }^{1} \mathrm{H}$. Schindler and J. Seelig, J. Chem. Phys, 59, 1841 (1973).

${ }^{2}$ E. Sackmann and H. Trăuble, J. Amer. Chem. Soc. 94, 4482 (1972).

${ }^{3}$ E. Sackmann and H. Träuble, J. Amer. Chem. Soc. 94, 4492 (1972).

${ }^{4}$ H. Träuble and E. Sackmann, J. Amer. Chem. Soc. 94, 4499 (1972).

${ }^{5}$ P. Devaux and H. M. McConnell, J. Amer. Chem. Soc. 94, 4475 (1972).

${ }^{6}$ C. J. Scandella, P. Devaux, and H. M. McConnell, Proc. Nat. Acad. Sci. 69, 2056 (1972).

${ }^{7}$ C. F. Polnaszek, G. V. Bruno, and J. H. Freed, J. Chem. Phys. 58, 3185 (1973).

${ }^{8}$ H. Shimizu, J. Chem. Phys. 37, 765 (1962).

${ }^{9}$ P. L. Nordio and P. Busolin, J. Chem. Phys, 55, 5485 (1971).

${ }^{10}$ G. R. Luckhurst, M. Seteka, and C. Zannoni, Mol. Phys. 28, 49 (1974).

${ }^{11}$ An order parameter $S=0.75$ is approximately the upper limit for the applicability of perturbation theory, since the correlation times of the spin probes dissolved in the smectic phase under investigation become larger than $3 \times 10^{-9} \mathrm{sec}$.

${ }^{12}$ H. Shimizu, J. Chem. Phys. 40, 754 (1964).

${ }^{13}$ R. E. D. McClung and D. Kivelson, J. Chem. Phys. 49, 3380 (1968).

${ }^{14}$ In the hydrodynamic approach (Debye-Perrin) the position of the gyration center does not affect the rotational diffusion. However the correction factor $k_{1}$ depends strongly on the locus of the center of gyration (cf. Ref. 12).

${ }^{15}$ R. T. Roberts, Nature 242, 348 (1973).

${ }^{16} \mathrm{~J}$. Charvolin and P. Rigny, J. Chem. Phys. 58, 3999 (1973).

${ }^{17}$ B. J. Gaffney and H. M. McConnell, J. Magn. Reson. (to be published). 